Extrême-Orient Extrême-Occident

\section{Extrême-Orient Extrême-Occident}

$36 \mid 2013$

Mobilité humaine et circulation des savoirs techniques (XVII ${ }^{\mathrm{e}}$-XIX ${ }^{\mathrm{e}}$ siècles)

\title{
La loupe et le miroir
}

The Magnifying Glass and the Mirror

微觀與對照

\section{Christian Jacob}

\section{(2) OpenEdition}

\section{Journals}

Édition électronique

URL : http://journals.openedition.org/extremeorient/317

DOI : 10.4000/extremeorient.317

ISSN : 2108-7105

\section{Éditeur}

Presses universitaires de Vincennes

\section{Édition imprimée}

Date de publication : 31 décembre 2013

Pagination : 211-217

ISBN : 978-2-84292-404-1

ISSN : 0754-5010

\section{Référence électronique}

Christian Jacob, « La loupe et le miroir », Extrême-Orient Extrême-Occident [En ligne], 36 | 2013, mis en ligne le 01 juin 2017, consulté le 03 mai 2019. URL : http://journals.openedition.org/extremeorient/317 ; DOI : 10.4000/extremeorient.317 


\title{
La loupe et le miroir
}

\author{
Christian Jacob
}

Au fond, quel est l'objet d'une histoire des savoirs? Qu'implique un tel projet, tant pour la démarche historienne elle-même que pour les questions qu'elle choisit de traiter?

Les textes réunis dans ce volume ont sur le lecteur non spécialiste que je suis un double effet: ils m'invitent à découvrir des situations, des moments, des acteurs dans des contextes culturels et historiques éloignés de mon terrain de compétence, ils les soumettent donc à l'éclairage de l'exotisme, de l'altérité, entre Chine, Japon et Corée. Mais d'un autre côté, ces études savantes ont aussi un effet de loupe, qui concentre l'attention sur un nœud de questions fondamentales, centrales. Je voudrais dans ces quelques pages tenter de rassembler et de formuler certaines de ces questions.

- Où résident les savoirs? Nous trouvons des savoirs incorporés et inscrits dans des personnes: il peut s'agir d'individus, ou d'un groupe d'individus identifiés par un statut social et professionnel, il peut s'agir également d'institutions; mais ces savoirs peuvent aussi être inscrits dans des textes ou d'autres dispositifs graphiques, et ces textes peuvent être mis en circulation. Il convient de prendre le temps de réfléchir à ces différentes catégories. Savoirs incorporés: ils définissent une qualité particulière d'un individu ou d'un groupe d'individus, une compétence. Celle-ci peut rester latente, virtuelle. $\mathrm{Ou}$ au contraire se manifester dans un savoir-faire particulier, par exemple un savoir technique, mobilisant des gestes et des instruments, savoir-faire dont on peut évaluer la performativité, les résultats, qu'il s'agisse de guérir un malade ou de penser un processus industriel innovant. Elle peut aussi se manifester dans une forme discursive, un certain type de compétence oratoire donnant à voir l'étendue des savoirs, mais aussi les raisonnements, les opérations logiques, la mémoire, l'aptitude à résoudre des problèmes ou à répondre à des questions. Ce savoir, cette compétence peuvent être les qualités propres d'un individu, ou caractériser un groupe social ou professionnel: les médecins, 
les astronomes, les lettrés, les mathématiciens, les ingénieurs. Ces collectifs peuvent être institutionnalisés et investis d'une autorité particulière. Quant aux savoirs objectivés, dans un texte écrit, une carte, des schémas, des tables, ils présupposent que la compétence particulière d'un individu ou d'un groupe peut être inscrite sur un support extérieur et matériel, qui permettra de conserver ce savoir ou de le transmettre à un tiers, par le biais des opérations spécifiques liées à la lecture, à l'interprétation et à l'apprentissage. Cette objectivation peut être régie par différentes contraintes: les savoirs peuvent être dépendants d'une langue ou d'un registre particulier de cette langue, d'une écriture, d'une forme discursive, d'un support matériel. La transitivité des savoirs inscrits de l'une à l'autre de ces modalités d'inscription dépend des institutions, de la politique des savoirs, de l'autonomie des textes à l'égard des explications orales, des instruments et de la bibliothèque, et de la mise en place de relais de transmission et de traduction d'une culture et d'une société à l'autre.

- Les savoirs comme objets et enjeux sociaux. Les savoirs deviennent tels à partir du moment où ils entrent dans des formes d'interaction sociale. Il y a d'un côté ceux qui les produisent et les détiennent, de l'autre ceux qui désirent les acquérir. Il y a ainsi une économie et une politique des savoirs. Ceux-ci sont investis d'une valeur particulière, valeur pratique, technique, symbolique, spirituelle, culturelle. Cette valeur ne réside pas seulement dans les formes d'application pratique des savoirs, dans ce qu'ils permettent de produire, mais aussi dans les qualifications qu'ils apportent à celui qui les détient, en termes de positionnement social et institutionnel, en termes de stratégies de carrière et de prestige personnel. Les savoirs assignent un lieu à qui les maîtrise, et le désir d'apprendre porte aussi bien sur le contenu et les effets des savoirs que sur ce lieu. Cette économie des savoirs peut prendre différentes formes. Il est des savoirs qui peuvent être partagés, distribués et communiqués sans restriction: dans ce processus, ces savoirs ne perdent pas leur valeur propre, ils ne sont pas dévalués en devenant publics. Leur maîtrise partagée, validée par des épreuves qualifiantes, est signe d'appartenance à une communauté, à une classe sociale, à une tradition culturelle. En revanche, il est des savoirs dont la valeur se fonde sur la rareté, sur la rétention, sur le monopole d'un milieu savant: ils sont alors les instruments de relations dissymétriques, un enjeu de pouvoir, un objet de négociation. Entre ces deux pôles se déploie un arc de situations où les savoirs font l'objet d'échanges, de transactions, de dons et de contre-dons. La valeur intrinsèque de ces savoirs peut elle-même varier, selon qu'ils sont marqués par la nouveauté ou par leur origine autorisée (la science et la technique occidentales, l'astronomie impériale chinoise...), par leur efficacité, par leur application pratique et leurs effets, par exemple thérapeutiques, par leur ancrage dans une tradition. Les savoirs les plus modernes, les plus à jour, les 
mieux actualisés peuvent faire l'objet d'une rétention institutionnelle, comme dans le cas de l'astronomie chinoise et de son application à l'établissement du calendrier. Pouvoir surmonter l'embargo sur les livres, sur les instruments, sur les savoir-faire suppose des stratégies complexes de contournement et de soumission, où les savants doivent cultiver les vertus de la diplomatie et parfois de l'espionnage. Don, contre-don, transaction : la vie sociale des savoirs déploie un véritable "marché des changes», où la valeur des connaissances, des livres, des calendriers, des savoir-faire pratiques se renégocie en fonction des aléas de la vie politique des états concernés.

- La morphologie des savoirs et leur plasticité. Les savoirs n'existent pas indépendamment d'une forme qui les construit, les fixe, les met en œuvre et les déploie. Cette forme peut être une séquence de gestes, un discours oral, un texte écrit, mais aussi différents diagrammes ou schémas. L'histoire des savoirs, et en particulier l'histoire de leur circulation sociale, doit prendre en compte les modalités de cette mise en forme et les marges de plasticité et de modification qu'elle peut subir sans que ses contenus s'en trouvent dénaturés. Les savoirs peuvent être attachés à une forme discursive particulière, à la littéralité d'un texte et à sa langue d'origine, à un ordre d'exposition précis. Inversement, dans certaines situations, ils peuvent être reformulables, traductibles, réinscriptibles, sans rien perdre de leur efficacité. Ici encore, nous nous trouvons face à un arc de possibles délimité par deux pôles. Particulièrement intéressantes sont les situations où des difficultés de compréhension linguistique conduisent à utiliser d'autres codes de communication, impliquant la traduction, mais aussi une grande déperdition et schématisation des savoirs concernés. Comment enseigner les sciences occidentales modernes en anglais à des élèves japonais? Comment se faire expliquer le mode de constitution des calendriers chinois lorsqu'on parle coréen et dans quelle mesure l'usage des signes d'écriture permet-il un niveau de communication translinguistique? La traduction des savoirs ne concerne pas seulement la lettre, mais aussi les cadres de référence idéologiques et spirituels : comment transposer les savoirs occidentaux, marqués par le christianisme, dans l'univers confucianiste ou néo-confucianiste? Et inversement.

- Il faut qu'un savoir soit ouvert ou fermé. Par «savoir fermé», j'entends un savoir complet, autonome, qui peut être transféré d'un bloc. Par «savoir ouvert», j'entends un savoir qui se prête aux corrections, aux ajouts, mais aussi aux suppressions, à une évolution cumulative, et parfois aussi à une révolution structurelle. Les savoirs «ouverts» intègreraient leur propre historicité, leur devenir, leur évolution, leur actualisation. L'ouverture et la clôture ne dépendent pas seulement de la nature des savoirs concernés, mais des dispositifs matériels ou discursifs dans lesquels ils sont fixés, qui 
peuvent ou non faciliter, ou simplement rendre possibles les mises à jour, les compléments, les corrections. Les savoirs «fermés» peuvent relever d'une stratégie particulière de communication et de transmission, sous la forme de «boîtes noires» dans lesquelles les savoirs sont encodés sans que leurs destinataires disposent des outils pour les modifier ou les actualiser. Le cas de l'établissement des calendriers chinois est particulièrement intéressant, dans la mesure où le calendrier lui-même n'explicite pas les savoirs et les règles de sa construction, des calculs qu'il présuppose. De plus, la mise à jour permanente de ces principes de construction, selon des savoirs eux-mêmes importés, l'astronomie européenne enseignée et traduite par les jésuites, rend les utilisateurs de cette technologie de comput du temps tributaires du bon vouloir de ceux qui en maîtrisent tous les arcanes. Les études réunies dans ce recueil soulignent les stratégies différentes d'explicitation des savoirs et de leurs principes, qui sous-tendent des stratégies de pouvoir et de monopole institutionnel. Je pense à un parallèle dans le monde contemporain: les logiciels «propriétaires », qui imposent régulièrement à leurs utilisateurs des updates et des upgrades payants; les logiciels «libres» ou open source, dont la mise à jour et les évolutions peuvent être réalisées par des communautés de développeurs décentralisées.

- L'histoire des savoirs comme genre discursif et ses différentes échelles. Les textes ici réunis relèvent tous de la micro-histoire: même s'il s'agit d'éclairer des phénomènes plus généraux, les transferts de techniques et de savoirs entre Occident et Extrême-Orient et aussi entre pays d'ExtrêmeOrient, l'accent est mis sur des personnages particuliers, sur leur circulation et leur œuvre propres, sur leurs réseaux et leurs sphères d'activités. Parmi les différents registres narratifs convoqués, la biographie occupe une place notable: comment situer l'activité intellectuelle, savante, scientifique dans une trajectoire de vie, avec ses facettes familiales, sociales, professionnelles, son déroulement linéaire, de l'enfance et des années de formation à la maturité? Une même enquête, du reste, peut croiser les points de vue et les perspectives, entre les différents acteurs et institutions impliqués. On retrouve la dimension interactive et sociale des savoirs, qui n'existent que dans la négociation, l'échange, la tractation, la conversion. Le choix de la micro-histoire permet également des jeux de décentrement, en se focalisant sur des acteurs secondaires, voire marginaux, dont la formation et l'activité se situent à l'écart des grandes institutions savantes. La micro-histoire met au premier plan de l'enquête des expériences localisées et datées, centrées sur un individu, un lieu ou un milieu: ces expériences introduisent de la complexité et la singularité de leurs scénarios dans la compréhension de phénomènes plus vastes, qu'il s'agisse des transferts de savoirs ou des hybridations entre savoirs locaux et savoirs 
étrangers, des rapports entre innovation et tradition. Cette pratique de la microhistoire, dans différentes aires culturelles et à différents moments historiques, met aussi en lumière la diversité des stratégies narratives et interprétatives résultant de la disparité même des sources, tant sur le plan quantitatif que sur le plan qualitatif. Qu'il s'agisse d'archives, de correspondances, d'annales, de récits autobiographiques, de textes officiels, d'écrits savants, les sources apportent autant des informations factuelles que des points de vue, partiels, biaisés, orientés, dont les angles morts sont aussi suggestifs que les données explicites.

- Lieux de savoir, espaces savants. Dans chacune de ces études, nous nous trouvons face à une pluralité de lieux et d'espaces. Il y a d'abord des lieux de savoir génériques, l'école, l'université, l'usine, qui définissent des sphères d'activités particulières - enseigner, apprendre -, reposant aussi sur le partage de rôles sociaux et institutionnels. Il y a ensuite des lieux géographiques, qui ne sont jamais isolés, mais prennent place sur une carte, dans un réseau de relations se déployant à différentes échelles, locales, régionales, nationales, transnationales. Ces relations sont modalisées et sémantisées par un ensemble de variables, relevant de la géographie physique et humaine, du positionnement des lieux par rapport aux centres de pouvoir et de savoir, de la hiérarchie et des rapports de concurrence entre ces lieux, mais aussi du réseau des voies de communication, de l'articulation du temps et de l'espace qui éloigne ou rapproche des lieux et en détermine l'accessibilité. Le voyage terrestre ou maritime et ses échelles temporelles, entre Occident et Extrême-Orient, mais aussi entre Chine, Corée et Japon, et à l'intérieur même de ces différents territoires imposent leurs rythmes propres, leur durée, leurs sas successifs au transfert des savoirs, des livres et des hommes qui en sont détenteurs. Les idées, les techniques, les innovations, la modernité se diffusent selon les rythmes multiples des moyens de communication et des vecteurs matériels, qu'il s'agisse de la correspondance, des revues, des livres, ou des savants euxmêmes. Dans cette géographie, les catégories de centre et de périphérie sont certes opératoires, mais demandent à être mises en abyme, à être redéfinies au fil de changement d'échelles successifs où les lieux les plus excentrés deviennent eux-mêmes les pôles de nouvelles constellations et jouent à leur tour un rôle dans la diffusion des savoirs.

- Possibilité d'une histoire croisée ou parallèle des savoirs, statut de la comparaison. Les contributions réunies dans ce volume s'inscrivent dans le renouveau d'une histoire des sciences et des savoirs transnationale, qui s'intéresse aux transferts et aux points de contact plus qu'à la problématique classique de l'antériorité ou des influences. De ce point de vue, un nouvel objet historiographique et anthropologique émerge: les lieux, la temporalité, 
les acteurs des transferts de savoirs ou de technologies et les phases de négociation comme les sas de traduction et d'acculturation qui permettent ces opérations. Une telle approche éclaire comme un entre-deux ou un no man's land des histoires nationales des sciences, et propose aussi différentes échelles d'observation: non seulement la confrontation de l'Occident et de l'Orient, mais aussi les interrelations multiples entre États et cultures de l'Extrême-Orient et, à l'intérieur de chacun de ces territoires, entre les centres de pouvoir et de savoir et les lieux périphériques. Dans ce paysage historiographique, déployé de manière très convaincante et suggestive dans cette livraison d'Extrême-Orient, Extrême-Occident, une perspective me semble particulièrement prometteuse: une histoire croisée des modes de rationalité, des styles intellectuels et des cadres épistémologiques. Il s'agirait moins de revenir à une histoire comparée des idées qu'à une histoire de leurs vecteurs, de leur mise en forme, de leur architectonique discursive et logique, de leurs fondations culturelles. Il s'agirait ainsi de cerner ce qui résiste au transfert, à la compréhension, à l'appropriation, d'une culture et d'une langue à l'autre, la part d'«intraduisible» dans les savoirs, et d'observer les stratégies de contournement, de déplacement, de «bricolage» mises en œuvre pour permettre la circulation de ces savoirs, au moins à un certain niveau. L'échelle d'une telle enquête ne serait pas les grands mouvements de transferts culturels, d'une aire à l'autre, mais les situations localisées, singulières, circonscrites à des individus et des groupes particuliers. La micro-histoire me semble être la bonne échelle pour comprendre ces jeux à l'intérieur de cadres prédéterminés, jeux avec les traditions, avec les langues, avec les concepts, avec le sens des mots, avec les savoir-faire. Dans cette histoire croisée, l'enjeu est non seulement de comprendre comment des savoirs et des savoir-faire peuvent être importés, traduits, adaptés, mais aussi d'observer ce que ces transferts produisent sur les détenteurs mêmes de ces savoirs et sur leurs savoirs euxmêmes: d'une certaine manière, il s'agirait d'étudier les effets de feedback et de rétroaction du transfert sur les cultures savantes dominantes.

L'histoire des savoirs est un champ en devenir, qui croise de multiples perspectives, de multiples outils intellectuels. Ce n'est que par des études de cas situées, écrites par des spécialistes des sociétés et des cultures concernées, que l'on peut aiguiser les questionnements et construire de nouveaux objets. La richesse des matériaux empiriques réunis dans ces études et les multiples jeux de miroir qu'elles créent entre différents mondes savants invitent à réfléchir sur les enjeux historiographiques et épistémologiques de l'étude des sciences, des savoirs et des techniques, de leur ancrage et de leur circulation dans l'espace. 
Ces quelques notes n'ont pas d'autre prétention que d'ouvrir les perspectives de réflexion, les questionnements que m'ont suggérés les études réunies par Catherine Jami. 